\title{
Les innovations dans l'enseignement des sciences, des mathématiques et de la technologie
}

\section{Edwyn James}

Traducteur : Patricia Vrinat

\section{OpenEdition \\ Journals}

Édition électronique

URL : http://journals.openedition.org/ries/3340

DOI : $10.4000 /$ ries.3340

ISSN : 2261-4265

Éditeur

Centre international d'études pédagogiques

\section{Édition imprimée}

Date de publication : 1 juin 1997

Pagination : 41-52

ISSN : 1254-4590

Référence électronique

Edwyn James, «Les innovations dans l'enseignement des sciences, des mathématiques et de la technologie », Revue internationale d'éducation de Sèvres [En ligne], 14 | 1997, mis en ligne le 30 juillet 2013, consulté le 14 novembre 2019. URL : http://journals.openedition.org/ries/3340 ; DOI : 10.4000/ ries.3340

Ce document a été généré automatiquement le 14 novembre 2019.

(c) Tous droits réservés 


\title{
Les innovations dans
}

\section{l'enseignement des sciences, des mathématiques et de la technologie}

\author{
Edwyn James
}

Traduction : Patricia Vrinat

1 L'Organisation pour la coopération et le développement économique (OCDE) a initié un projet sur la réforme des curricula et l'efficacité de l'école en 1987, ce qui a donné lieu à deux initiatives importantes. L'une d'entre elles passait en revue les tendances dans la réforme des curricula ${ }^{2}$ et l'autre concernait l'établissement de certains curricula de développement, y compris une étude internationale des innovations dans l'enseignement des sciences, des mathématiques et de la technologie $\left(\mathrm{SMTE}^{3}\right)$. On a montré beaucoup d'intérêt pour cette étude dans de nombreux pays, car on considérait ces matières vitales pour le bien-être et la prospérité futurs, mais, en même temps, on était préoccupé par leur impopularité apparente parmi la jeune génération. On peut également noter qu'en général on reconnaît deux autres arguments de poids en faveur de l'importance de cette étude. Le premier est le droit intrinsèque de tous les jeunes de ne pas être exclus de ce vaste domaine de connaissances humaines qui croît sans cesse, mais, au contraire, d'y avoir accès à un niveau qui correspond au propre potentiel de chacun. Le deuxième est le besoin de chaque futur citoyen d'avoir pu user de ce droit pour garantir que, dans une société démocratique, les décisions prises sont informées et responsables.

2 Cependant, la vitesse de changement dans notre société déjà complexe est telle, et plus particulièrement en sciences, en mathématiques et en technologie, que nous devons insister encore plus sur la nécessité de répondre aux besoins et aux droits des individus. Chacun doit être doté de capacités qui permettront de faire en sorte que l'apprentissage, tout au long de la vie, soit une réalité. Pour aller de pair avec de telles considérations, le travail de préparation pour l'étude a établi des objectifs de 
changement très larges, qui devraient rendre le SMTE plus attractif et pertinent pour chacun:

- on a pris en compte des considérations sociales et environnementales puisque, sans reconnaissance suffisante de ces aspects, on ne pourrait pas susciter ni entretenir l'intérêt des jeunes ;

- on a considéré les élèves, non seulement comme membres d'un groupe, mais comme des individus avec leurs propres besoins et leurs propres droits, y compris le droit à une éducation qui correspond le mieux possible à ces besoins.

De plus, on a reconnu que l'éducation n'était pas la chasse gardée des experts et que l'on doit plutôt la considérer comme une entreprise qui demande le soutien actif de chaque élément de la société. Dans une société donnée, toute école dépend de cette société et a des responsabilités envers elle.

Il n'a jamais été question de faire une étude d'ensemble des pratiques contemporaines, mais plutôt de chercher à identifier des innovations intéressantes et significatives dans le domaine du SMTE, qui pourraient former la base d'une étude de cas avec un cadre thématique défini. Il ne s'agit pas d'insister sur la méthodologie de l'étude de cas employée, qui n'a rien d'original en soi, mais de passer en revue les résultats de ces études et de leur comparaison entre elles qui a suivi, de voir l'éclairage qu'ils donnent à la recherche d'un moyen de développer pour chacun des aptitudes scientifiques et techniques.

On a préparé des propositions préliminaires pour des études de cas dans de nombreux pays membres et on a publié huit études exploratoires qui viennent des États-Unis ${ }^{4}$. La réalisation des études de cas, qui avait commencé en 1991, ou même avant, s'est étendue et, dans les deux années qui ont suivi, il y a eu une collaboration internationale importante qui faisait appel à vingt-trois groupes de recherche dans treize pays. À l'intérieur de ces groupes, on a constaté une variété d'approches nouvelles de l'enseignement et de l'apprentissage des sciences, des mathématiques et de la technologie. Par le biais de réunions de travail internationales et d'autres échanges organisés par le Secrétariat de l'OCDE, on a identifié des traits communs. Ainsi, les groupes de recherche ont gardé un lien entre eux et ils ont développé des similarités dans leurs approches. Cette entraide mutuelle a été bénéfique pour l'étude en général. Chaque groupe a remis son rapport au Secrétariat en août 1995 et, par la suite, un rapport de synthèse a été préparé ${ }^{5}$. Les membres de l'équipe internationale chargée de rédiger ce rapport avaient aussi participé à l'élaboration de cette étude depuis le début.

6 Ce rapport de synthèse contient un grand nombre d'informations sur les tendances de l'enseignement dans ces matières prises soit individuellement soit dans leur ensemble et met en évidence quatre points qui seront examinés par la suite :

- l'autonomie plus grande de l'apprenant;

- l'accent mis sur l'apprentissage par la pratique et l'expérience ;

- l'intégration des matières;

- l'emploi très étendu de la technologie de l'information.

7 Toutes ces études permettent de comparer des situations différentes. Parfois les innovations ont reçu un accueil favorable et ont été mises en place avec succès ; parfois il y avait un conflit évident entre les objectifs de réforme visés et les résultats mitigés obtenus. Si on veut être sûr de fournir une éducation en sciences qui soit plus attractive, plus globale et plus efficace, il faut prendre en compte certains points qui 
ont paru importants pour la mise en place réussie de nouveaux curricula. On peut citer les points suivants :

- l'établissement de liens étroits entre l'école et la communauté ;

- le professionnalisme de l'enseignant et les changements dans son rôle.

\section{Les études de cas}

8 Chacune des vingt-trois études de cas est introduite brièvement pour expliquer comment elle peut influencer l'enseignement en sciences, en mathématiques et en technologie et, ensuite, les études menées en Australie et en Allemagne sont traitées de façon plus détaillées, à titre d'illustration. Outre les études venant des treize pays concernés par la recherche, une grande conférence de diffusion sur ce travail se tiendra dans le courant de l'année 1997, au Mexique, pays qui a commencé à s'intéresser au SMTE, après la phase d'élaboration. Pour les pays d'Amérique latine, cette conférence sera une excellente occasion de mettre en pratique les résultats de l'étude dans une situation où l'on cherche, de toute urgence, une éducation de qualité pour tous.

9 La diversité des études de cas est frappante, pas seulement par leur étendue et la quantité de ressources à mettre en œuvre, mais aussi par le fait qu'elles partageaient toutes le souci commun d'améliorer la qualité et l'efficacité de l'éducation fournie ${ }^{6}$.

Dans un cas extrême, une étude de cas en Colombie-Britannique, au Canada, ne concernait qu'une seule salle de classe et un seul aspect de l'enseignement de la physique. Cette étude favorisait l'égalité entre les deux sexes avec un certain succès, puisque les élèves étaient eux-mêmes impliqués et élaboraient leur propre cours pour pouvoir incorporer des préoccupations sociales. On pense que les stéréotypes liés au sexe sont le résultat d'interactions complexes entre l'école et la communauté et ont pour conséquence que les filles sont souvent exclues du SMTE (ou, du moins, ont l'impression de l'être). Ailleurs, en Norvège, trois classes de sciences de niveau seconde ont été suivies pendant un trimestre; il s'agissait de l'élaboration et de la mise en pratique d'un cours sur l'électricité, centré sur les élèves et qui se terminait par une épreuve orale innovatrice. L'étude, en Irlande, fait un compte rendu d'une initiative gouvernementale axée sur la mise en place de l'enseignement de la physique et de la chimie de 15 à 18 ans, dans trente-trois écoles (soit de filles, soit mixtes), où il n'y avait pas de tradition de l'enseignement de ces matières dans le passé. Des professeurs expérimentés dans ces disciplines ont été en partie détachés pour enseigner dans ces écoles où les professeurs qui prendraient leur suite ont pu les observer; de telles associations étaient efficaces, satisfaisantes sur le plan professionnel pour les deux parties concernées et réussies dans la mesure où il y avait une augmentation considérable du choix de ces disciplines par les filles. Sur une plus grande échelle, mais encore une fois pour rendre une seule matière plus populaire, la Société américaine de chimie a sponsorisé La Chimie dans la vie quotidienne qui insiste sur la pertinence et l'intérêt social de la matière ; la première publication eut lieu en 1988 et, en septembre 1991, jusqu'à 250000 élèves des high schools avaient appris la chimie selon cette méthode. Ces exemples très brefs indiquent :

- une extension des activités professionnelles des enseignants ;

- un changement dans les relations entre élèves ;

- une mise en place d'actions efficaces pour rendre les sciences plus attractives. 


\section{L'intégration des matières}

11 Souvent, en sciences, on voit une intégration de la physique, de la chimie et de la biologie et parfois des sciences de la terre, d'autres domaines étant également incorporés. L'élan pour une telle intégration est venu de l'envie de rapprocher la science à l'école des pratiques des scientifiques contemporains et des ingénieurs. Ainsi, par exemple, les développements récents en génétique ou dans les transplantations chirurgicales, dans les communications ou dans l'agriculture intensive, ne peuvent être délimités par les frontières traditionnelles entre les matières, mais font appel à des éléments divers comme l'éthique ou l'économie. Les sciences à l'école doivent connaître ces changements et les prendre en considération.

12 En accord avec de telles notions, le nouveau curriculum des sciences au Japon (pour les 9/15 ans) est focalisé sur la responsabilité humaine, l'individualité, l'ingéniosité et vise clairement à stimuler la créativité plutôt que l'acceptation passive de faits. Un aspect particulier est l'élaboration et la mise au point d'un plan de cours au sein d'une équipe de professeurs. Un travail qui a pour origine des préoccupations contemporaines, comme les chandelles de béton (un phénomène de l'érosion du béton causé par l'acidité atmosphérique), va nécessairement au-delà des interprétations traditionnelles des matières. Comme les enseignants japonais l'ont dit dans leur rapport d'étude sur leurs élèves en sciences :

«Traiter des matériaux généraux en cours de sciences n'intéresse pas les élèves... Ils sont intéressés par leur environnement. Les élèves commencent à trouver une relation entre leur environnement proche et eux-mêmes. De plus, les élèves ont commencé à apprécier la nature. »

En Ontario, au Canada, l'étude de cas examine une situation où il y a un changement radical en neuvième classe où des spécifications nationales nouvellement introduites visent à l'intégration des cours de sciences et de mathématiques dans des classes hétérogènes, avec des objectifs d'apprentissage bien spécifiques. L'étendue de ce changement a créé des problèmes, à la fois aux enseignants et aux élèves. L'Espagne a adopté un curriculum de sciences intégrées qui accompagne la prolongation de la scolarité obligatoire jusqu'à 16 ans; l'étude de cas cherche à voir comment le curriculum se met en place à travers des activités communes et comment les enseignants veulent donner des responsabilités aux élèves. L'Association américaine pour la promotion des sciences (qui n'est pas d'origine gouvernementale) a établi un projet de réforme nationale des curricula en sciences; elle a adopté une approche intégrée pour donner des compétences scientifiques à tous. Les résultats ont été étudiés dans six districts scolaires, pour voir les conséquences sur l'élaboration des curricula, les tensions éventuelles et pour étudier ce qui a été accompli. On est d'accord pour dire que moins, c'est mieux; c'est-à-dire que couvrir un champ de connaissances moins large mais de manière moins superficielle améliore l'éducation. Un autre exemple américain concerne la mise en place d'un cadre pour un curriculum détaillé en Californie et indique que, même si des échanges entre professeurs ont conforté l'engagement de réformer, la qualité de l'enseignement des sciences et sa corrélation avec un cadre établi varie considérablement. L'argument en faveur d'un enseignement intégré des sciences semble comporter deux volets :

- les frontières traditionnelles entre les différentes sciences ne correspondent pas aux expériences de la vie contemporaine; 
- un élargissement de l'opinion positive vis-à-vis des sciences et un plus grand intérêt parmi les jeunes seraient probablement favorisés par une approche intégrée à l'école.

\section{Travailler ensemble} peut être introduite avec succès à un moment donné, comme dans les exemples précités en Ontario et en Espagne. Ces études soulignent aussi la nécessité de former des associations et des réseaux d'échanges pour un meilleur partage des responsabilités. Des enseignants isolés ne peuvent pas mettre en place efficacement de nouvelles idées de curricula imposées par une source extérieure, surtout quand ces idées dépassent leur compétence dans la discipline ou dans la pédagogie à employer. Il leur faut du temps pour s'adapter à des implications nouvelles et pour remettre de l'ordre dans leur approche. De même, il faut le dire, les enseignants n'ont pas la compétence nécessaire, ni le droit d'agir seuls, coupés des autres participants du monde éducatif qui devraient exercer leurs responsabilités, être partie prenante et que l'on doit consulter. Il faut associer tous les groupes d'intérêt sur une base de respect mutuel et de coopération. Sur une telle base, les écoles peuvent profiter des services des spécialistes et des entreprises de la société, comme les musées ou les centres scientifiques interactifs, en utilisant leurs expositions ou le service offert par leur personnel.

L'étude australienne (en Tasmanie) fournit un exemple intéressant de partage de responsabilités. Des déclarations nationales et des projets de curriculum ont été examinés par chaque État ; par la suite, chaque État a créé sa propre grille curriculaire. Même après tout ce travail, on a laissé beaucoup de liberté à chaque école sur le plan local. On peut noter que tout le personnel d'une des écoles de l'étude de cas a choisi de concentrer son expérimentation professionnelle seulement sur les mathématiques, même si l'État avait demandé d'agir aussi en sciences, en technologie et en alphabétisation. Le personnel a eu le sentiment qu'il y avait plus à gagner à se concentrer sur un domaine, puisqu'il leur manquait du temps pour tout faire. On a encouragé une telle liberté d'action dans les écoles; il s'agit de l'approche appelée opportunity within frameworks (l'opportunité dans un cadre spécifique). Une autre école a choisi d'utiliser les matériaux nationaux et ceux fournis par leur État pour procéder à leur propre vision des mathématiques, au cours d'une période de deux années et, ainsi, obtenir une juste appréciation de son potentiel. Les échanges entre professeurs sont allés jusqu'à enseigner en équipe, avec deux ou trois professeurs partageant la responsabilité d'une classe, au moins en partie, et avec des réunions régulières pour l'élaboration du curriculum.

Très souvent, les écoles en Tasmanie ont souligné l'importance d'un élément clé : un professeur compétent et expérimenté, soutenu par le personnel de direction dans l'école même et par les responsables de l'éducation en dehors de l'école. Les parents se sont investis aussi dans les écoles, ils ont participé à des ateliers de travail pour être parfaitement bien informés des évolutions et pour donner leur soutien. On a reconnu que tous ceux qui sont concernés par le système éducatif devaient participer à la prise de décisions et qu'il fallait une convergence de points de vue. On ne peut pas réussir à mettre en place des changements par simple décret. 
17 Le curriculum innovant de sciences intégrées en Allemagne, pour les niveaux qui vont de la cinquième à la dixième classe (11-16 ans), est le fruit d'une collaboration qui a commencé quand l'Institut national allemand pour l'enseignement des sciences (IPN) a apporté son soutien à des enseignants du Schleswig-Holstein. Le projet souligne la relation entre l'humanité et la nature et s'est élargi volontairement, sur une grande échelle, à d'autres Länder. Comme dans beaucoup d'autres pays, l'enseignement des sciences en Allemagne est confronté à la nécessité d'attirer davantage d'élèves et de développer une base de connaissances qui prépare l'avenir mais qui reflète aussi la nature ambivalente des sciences modernes, que l'on considère moins comme une question de vérité absolue mais plutôt comme une question d'explications provisoires.

Les membres du projet Practising Basic Integrated Science in Germany (PING, c'est-à-dire un projet en faveur de la pratique des sciences intégrées en Allemagne) ont adopté un point de vue commun en ce qui concerne l'innovation, centré tout particulièrement sur l'enseignant. Les enseignants et les écoles veulent changer leurs modèles d'enseignement pour répondre aux besoins de tous les élèves dans le monde contemporain. Le rôle de l'institut de recherche est d'offrir des conseils, une certaine coordination et de développer les recherches. La participation des enseignants à PING est assurée par la collaboration et le développement permanent de matériaux appelés "matériaux de suggestion», qui sont une série de fiches de travail qui peuvent être adaptées localement, comme Les animaux et moi-même et qui sont une forte source de motivation pour les élèves. L'enseignant dans sa classe facilite l'emploi de ces matériaux :

«J'ai mis les matériaux sur la table, en disant «les voici», et j'ai demandé aux élèves de les examiner pour trouver des suggestions pour accomplir leur tâche. Ils ont beaucoup apprécié cette idée et je les ai aidés à coordonner leur travail. »

19 Puisque la participation des enseignants dans le travail de développement prend du temps, il s'avère qu'une collaboration avec l'administration gouvernementale locale pour l'éducation est essentielle. Pour le moment, PING est reconnu dans plusieurs régions comme un projet pilote national et, donc, les enseignants des régions concernées ont une décharge pour leur participation.

20 Dans le passé, les tentatives destinées à améliorer l'enseignement des sciences en Allemagne se sont limitées au développement des matériaux pour une seule matière, ciblées sur un groupe d'âge sélectionné ou sur des méthodes d'instruction spécifiques. Les membres de PING pensent que de telles innovations resteront isolées et ne correspondent pas à la réalité des besoins des écoles. Alors le projet a mis au point une progression de la cinquième à la dixième classe (11-16 ans), qui prend en compte le progrès individuel et social de chaque élève. PING soutient aussi une large gamme de méthodes épistémologiques qui encouragent les enseignants à essayer une variété de techniques centrées sur l'élève.

21 L'élan pour cette réforme est venu de nouvelles écoles uniques qui accueillent tous les élèves, dans la région du Schleswig-Holstein. Les enseignants voulaient promouvoir l'efficacité de ces écoles en introduisant un meilleur enseignement des bases en sciences. Comme le projet PING s'est révélé un succès, d'autres types d'écoles se sont joints au projet pilote. Avec PING, la relation entre les connaissances pédagogiques, l'intégration et les disciplines scientifiques traitées séparément, est toujours prise en considération dans le choix des matériaux, surtout pour les classes accueillant des élèves de 11 à 16 ans. La recherche sur les attitudes des élèves a montré que, lorsque la 
conception PING est mise en œuvre, ils apprennent mieux, ils ont plus de satisfactions et les sciences sont plus attractives.

Ceci est donc une innovation où :

- il y a une association active entre enseignants en milieu scolaire et enseignants/chercheurs universitaires ;

- les fondements des curricula sont présents dans la vie et l'expérience de tous les jours;

- on a aidé les enseignants pour qu'ils puissent assumer une charge de travail plus lourde ;

- la mise en place dans d'autres régions s'est faite de façon volontaire;

- les élèves montrent un plus grand intérêt et une plus grande motivation en sciences.

\section{Leçons à tirer des mathématiques}

Les études sur les mathématiques auxquelles on fait brièvement allusion ici ont des similitudes avec celles sur les sciences et la technologie ; par exemple, les enseignants participent à des activités communes. Sur le plan local, les enseignants américains et d'autres membres de la communauté qui les entoure ont travaillé ensemble avec succès, par la tenue régulière de réunions pour améliorer la qualité de l'enseignement des mathématiques et par la création de seize Urban Mathematics Collaboratives (des groupes de travail par ville). Cependant, il faut admettre que, même à l'intérieur des régions concernées, ces associations ne touchaient qu'une partie des écoles, ce qui indique, comme c'est souvent le cas, que la mise en œuvre du changement n'est ni simple ni facile et nécessite un engagement à long terme.

En France, on a introduit une évaluation nationale pour les élèves âgés de 8,11 et 15 ans, pour que les enseignants de mathématiques puissent mieux identifier les besoins de leurs élèves en début d'année scolaire et adapter leur approche en conséquence. Cette étude souligne les bénéfices d'un dialogue entre enseignants et parents et montre que l'on souhaite davantage de formation en cours de carrière. On peut remarquer qu'il y a eu peu de travail sur l'évaluation en sciences et en technologie dans les études SMTE. Que ce soit sous la forme de tests de diagnostics ou de l'autoévaluation ces exemples donnent des modèles intéressants pour le contexte d'autres curricula et suggèrent qu'il est possible d'avoir une expérience plus enrichissante et plus significative pour les étudiants.

Les écoles japonaises ont introduit un curriculum révisé de mathématiques et ont réduit le temps d'enseignement disponible. Dans le rapport de cette étude, on retrouve des éléments communs à beaucoup de projets en sciences et le désir de mettre l'accent sur la résolution de problèmes et sur le travail individuel, qui tiennent compte des progrès en technologie. Une autre approche nationale (mais non gouvernementale) pour améliorer l'enseignement des mathématiques a été étudiée aux États-Unis, où le Conseil national des enseignants de mathématiques s'est mis d'accord sur certains points et a défini des critères d'exigences minimales (Standards) pour améliorer l'enseignement des mathématiques dans tout le pays. Le Conseil met l'accent sur la rédaction et la résolution des problèmes à l'aide de calculatrices et d'ordinateurs. Cependant, ce mouvement vers la définition de normes de performances, que ce soit en mathématiques ou en sciences, n'est pas bien accueilli partout aux États-Unis. On peut considérer qu'il est potentiellement en conflit avec les activités centrées sur l'individu, qui essaient de bien prendre en compte les circonstances locales et les besoins individuels. 


\section{L'emploi des ordinateurs}

On a déjà mentionné l'importance des ordinateurs dans ces développements de curricula. Une école aux États-Unis était à l'origine d'un cours d'initiation au calcul infinitésimal, que l'on a maintenant adopté très largement ailleurs dans le pays et qui est fondé sur la création de modèles de vrais phénomènes en utilisant des calculatrices graphiques et des ordinateurs. De même, l'étude suisse explique comment l'emploi d'ordinateurs peut être un moyen qui permet aux élèves de 13 à 16 ans de comprendre des phénomènes réels, comme la diffusion d'épidémies ou la vitesse de croissance d'une plante. En Autriche, le ministère a formé un petit groupe de professeurs expérimentés, pour créer des matériaux qui font appel aux ordinateurs pour l'enseignement des mathématiques dans des sections technologiques pour des élèves âgés de 15 à 19 ans ; on a constaté partout l'intérêt de tous, même si l'on savait que les exigences étaient plus grandes pour les élèves à cause de leur participation plus active. On a vu que la création de modèles de vrais phénomènes naturels peut élargir les frontières des sciences, et même de la technologie à l'école, pour englober des thèmes et des préoccupations qui sont d'un intérêt intrinsèque pour des jeunes. Les ordinateurs sont utiles non pas comme une fin en soi mais comme un moyen de traiter et de présenter les données. Leur emploi facilite l'adoption de curricula d'apprentissage qui sont fondés sur l'expérience, ce qui représente une inversion par rapport aux approches traditionnelles, plus théoriques, où l'on mentionnait brièvement, voire pas du tout, des applications pratiques, une fois que la théorie avait été totalement développée.

Pour un groupe d'âge plus jeune (9/14 ans), une étude aux États- Unis a utilisé des ordinateurs et des vidéos pour simuler une exploration (The Voyage of Mimi), permettant de développer des concepts en sciences et en mathématiques ainsi que dans d'autres domaines du curriculum. L'étude de cas indique comment les enseignants ont collaboré et comment ils ont adapté ces matériaux. La population scolaire était plus jeune encore (8/11 ans) pour un projet aux États-Unis intitulé Kids Network (Le réseau des enfants), qui a été créé pour générer des données locales sur des questions d'environnement et pour les partager avec d'autres centres par la communication électronique. Le rapport considère le projet comme un moyen de diversification pour les enseignants plutôt que comme un produit déterminé. Dans les deux cas cités, il faut se souvenir que, quelles que soient les intentions et les attentes de l'innovateur dans le curriculum, c'est dans la salle de classe que l'aspiration devient une réalité. Alors que la technologie électronique offre des possibilités pédagogiques considérables, l'enseignant a, et continuera à avoir, un rôle central dans l'interprétation donnée au curriculum et dans sa mise en pratique.

\section{Technologie}

Il est évident que la technologie électronique a elle-même donné l'élan à plusieurs des études entreprises, que ce soit en mathématiques, en sciences ou dans des activités pluridisciplinaires. Avec une approche différente, la technologie peut être une matière entièrement indépendante qui nécessite une étude à part. Donc, l'étude de cas des PaysBas parle de l'introduction de la technologie auprès d'élèves hollandais âgés de 12 à 14 ans, pour suivre une politique gouvernementale. Au départ, il y avait un conflit à 
résoudre entre l'apprentissage de capacités pratiques et les connaissances exigées par le curriculum. Il semblerait que les implications sociales de la technologie n'aient pas été bien prises en compte et il y avait peu de coopération avec les enseignants des matières scientifiques, malgré les similitudes avec les curricula. Les garçons semblaient davantage intéressés par cette matière que les filles.

Des directives nationales dans les écoles en Écosse, pour des élèves de 5 à 12 ans, ont aussi donné lieu à l'introduction de cette nouvelle matière, la technologie. On a identifié des personnes clés qui étaient indispensables pendant la mise en place pour conduire les changements, une communication très ouverte entre l'école et la communauté allant de pair. De même, en Tasmanie, un enseignant clé était nécessaire pour introduire la technologie et les changements en sciences et en mathématiques entre 4 et 16 ans, suite à des initiatives lancées par le gouvernement. L'étude en Tasmanie, encore plus que dans le passé, met l'accent sur l'apprentissage centré sur l'élève et le travail en commun entre professeurs. Il faut noter que très peu des vingttrois études s'occupaient directement de la technologie et qu'aucune ne traitait la question délicate de l'évaluation et de la progression. Il n'y a pas eu de consensus sur la question de savoir si la technologie devrait être une matière à part entière ou un élément qui découle des matières traitées par ailleurs dans le curriculum. Là où la technologie était traitée comme une matière indépendante, deux facteurs apparaissaient :

- des enseignants clés, le travail en commun entre les enseignants et une communication ouverte avec la communauté et les élèves étaient importants pour l'introduction réussie de la technologie ;

- mais l'équilibre souhaitable entre la connaissance et des capacités pratiques restait incertain, ainsi que le moyen de rendre la matière attrayante pour tout le monde.

La discussion ci-dessus couvre un domaine très vaste et beaucoup d'aspects nous incitent à continuer nos recherches pour créer une bonne compréhension des sciences dans la société. On a identifié des priorités qui sont à prendre en compte par les décideurs si on veut fournir une meilleure éducation en sciences à l'école, qui puisse être entretenue au cours de la vie adulte. Le système éducatif et les enseignants qui en font partie doivent avoir la possibilité de fournir le service le mieux adapté aux besoins de chaque élève.

De plus en plus, on doit considérer l'élève comme un individu responsable qui a droit à l'initiation au monde des sciences et de la technologie, un monde présenté de manière à susciter l'intérêt et l'investissement personnel. Il semblerait que ceci nécessite, très souvent, une approche intégrée, puisqu'elle a une relation directe avec les préoccupations et le mode de vie des jeunes d'aujourd'hui et avec les questions centrales de la vie contemporaine. Elle prend donc en considération le contexte actuel et la sensibilité aux problèmes environnementaux et sociaux. Cet accent mis sur l'apprentissage pratique et expérimental, dans un contexte intégré, minimise l'importance des frontières historiques entre des matières telles que la physique, la chimie ou la biologie, puisque ces frontières ne font pas partie de la réalité présente. Des difficultés peuvent survenir du fait que l'enseignant bien qualifié est probablement spécialiste d'une seule des disciplines du SMTE et, de ce fait, moins convaincant et moins charismatique quand il traite d'autres sujets. Cependant, l'enseignant moins qualifié, ou même peu qualifié, peut détourner l'élève de certaines disciplines au lieu de susciter son intérêt. Si l'on veut faire une réforme efficace pour l'élève, il faudra peut- 
être redistribuer des ressources déjà rares : l'inaction n'est autre qu'une confirmation de fait du statu quo, et n'est pas franchement défendable. Nous ne pouvons pas déplorer le manque d'intérêt des élèves sans remettre en cause la nature du curriculum qui leur est proposé, ni sans mettre en œuvre des mesures pour faire une réforme appropriée.

Le rôle de l'enseignant est essentiel dans toute action pour améliorer l'enseignement, non seulement dans l'exécution des réformes (ce qui est l'aboutissement du procédé), mais aussi au cours de leur planification et de leur élaboration. On voit les enseignants participer activement à l'élaboration des curricula et à leur évaluation; ils arrivent à bien intégrer la technologie électronique dans leur enseignement pour diversifier les outils pédagogiques employés, sans que cela ne devienne une contrainte; ils collaborent avec leurs collègues, avec les chercheurs universitaires et ceux qui prennent des décisions d'ordre politique. Ce partenariat implique une meilleure appréciation, plus ouverte, du professionnalisme de l'enseignant. On doit faire en sorte que les enseignants soient parfaitement équipés pour répondre aux exigences de ce nouveau rôle, entre autres, par la formation continue.

Le système éducatif doit répondre de manière cohérente à cette analyse des besoins des élèves et des professeurs et doit introduire les innovations qui conviennent. On parle souvent de réforme systémique, ce qui pour certains est une métaphore tirée de l'ingénierie mécanique, où le succès demande que chaque composant du système soit totalement opérationnel. Selon ce point de vue, un changement effectué dans une partie du système devrait entraîner des répercussions par ailleurs. La métaphore d'un écosystème serait peut-être plus prometteuse : on pourrait considérer l'innovation à l'école, ou dans une classe, comme une culture. Avec un environnement favorable, cette culture va se développer et même commencer une colonisation. L'étude SMTE a montré l'accroissement de l'intérêt et de l'implication quand des innovations se sont développées dans un environnement encourageant. Nous devons, pendant qu'il en est encore temps, comprendre la nécessité vitale de créer des conditions qui seront favorables au développement et à l'évolution d'une éducation de qualité, faute de quoi l'éducation risque d'être de plus en plus isolée de la population qu'elle cherche à servir.

\section{NOTES}

2. M. Skilbeck, Curriculum Reform: an Overview of Trends, Paris, OCDE, 1990.

3. L'étude SMTE constitue un des éléments d'une étude plus large qui est en cours : Les enseignants et la réforme des curricula dans l'enseignement de base.

4. OCDE, Science and Mathematics Education in the United States: Eight Innovations, Paris, OCDE, 1993.

5. P. J. Black et J. M. Atkin, Changing the Subject - Innovations in Science Mathematics and Technology Education, Londres, OCDE/Routledge, 1996.

6. D'autres renseignements sur chaque cas (y compris comment obtenir des copies des rapports) sont disponibles dans le rapport d'ensemble sur le SMTE in: Black et Atkin, op. cit. note 4). L'intégralité des huit études américaines est aussi publiée, avec une analyse de chacune d'entre elles dans une publication distincte: S. A. Raizen et E. Britton, Bold Ventures: US Innovations in Science and Mathematics Education, Boston, Kluwer Academic Publishers, 1996, 3 volumes. On peut 
obtenir un résumé de chacune des vingt-trois études sur le serveur de l'OCDE: http:// www.oecd.org/els/publicatio/edu/smte.htm

\section{RÉSUMÉS}

L'étude internationale coordonnée par l'OCDE présente vingt-trois innovations dans l'enseignement des sciences, des mathématiques et de la technologie. Bien que répondant à des politiques différentes et prenant des formes diverses, toutes ces actions mettent en évidence l'importance des liens entre l'école et son environnement, le professionnalisme nécessaire des enseignants ainsi que l'évolution de leur rôle.

\section{INDEX}

Mots-clés : enseignement technique, études scientifiques, innovation, mathématiques, programme d'enseignement, sciences

Index géographique : Pays de l'OCDE, Australie, États-Unis, Allemagne, Écosse, Royaume-Uni

\section{AUTEURS}

\section{EDWYN JAMES}

Consultant à l'OCDE 